\title{
Guided resonance in negative index photonic crystals: a new approach
}

\author{
Silvia Romano ${ }^{1}$, Stefano Cabrini ${ }^{2}$, Ivo Rendina ${ }^{1}$ and Vito Mocella ${ }^{1}$ \\ The behavior of a negative refraction photonic crystal slab irradiated with out-of-plane incident beam is an unexplored subject. In such \\ an experimental configuration, guided mode resonance appears in the reflection spectrum. We show that, in this case, the light coupled \\ inside the photonic crystal is backpropagating. A relationship with the negative index properties is established using a new approach in \\ which the guided resonance is recovered by modeling the photonic crystal layer with a simple Lorentz resonator using the Fresnel \\ reflection formula.
}

Light: Science \& Applications (2014) 3, e120; doi:10.1038/Isa.2014.1; published online 3 January 2014

Keywords: diffraction gratings; guided mode resonance; negative index; photonic crystals

\section{INTRODUCTION}

In 1902, Wood observed the presence of narrow bright and dark bands in the reflectivity spectrum of an optical grating. These bands were dependent on the polarization of the incident light, and because they could not be explained by grating theory, they were classified as anomalies. ${ }^{1}$ This effect was theoretically explained for the first time by Rayleigh $^{2}$ and then by Hessel and Oliner ${ }^{3}$ in 1965, who demonstrated that these anomalies in the reflections from gratings were related to the excitation of surface waves on metallic grating structures. Similar resonant anomalies have been observed in various materials with a periodic patterning applied to a surface that can support excitations, such as plasmon polariton resonance or sharp spectral features in shallow grating waveguide structures. ${ }^{4}$ In particular, the reflection and transmission of an incident wave on a photonic crystal $(\mathrm{PhC})$ slab can produce sharp resonance in the spectrum when the radiation is coupled with the modes of the structure. ${ }^{5-7}$ Guided mode resonance has been well studied in the photonic crystal literature. Due to the extremely narrow shape of the resonance when superposed on the background reflection, guided mode resonances can be used to design optical bandpass filters with elevated symmetrical responses and low side-bands ${ }^{8,9}$ or distributed feedback lasers with a high $Q$ factor. ${ }^{10}$ Moreover, the confinement of the optical field within the slab can be used to trap ${ }^{11}$ single particles or to enhance signals from fluorescent elements, thus enabling high-sensitivity sensors. ${ }^{12}$ In this paper, we reveal novel insight into the reflectivity properties of a negative refraction photonic crystal slab. In particular, by studying a hexagonal airhole lattice in silicon, we highlight that the out-of-plane incoming radiation is negatively refracted in the structure. While the in-plane properties of negative refraction in a photonic crystal slab have been studied over the last years, ${ }^{13-20}$ this is the first experimental demonstration of negative refraction detected out-of-plane. In particular, we provide imaging of the radiation coupled into a photonic crystal slab when the resonance occurs. Using an infrared camera, we were able to visualize the interaction between the incident light and backpropagating coupled radiation. In addition, we propose a new theoretical approach to the guided resonance phenomenon, based on the Fresnel formula, which shows very good agreement with the experimental data and which completes the usually adopted phenomenological model ${ }^{21-23}$ in the context of the Fano resonance approach.

\section{MATERIALS AND METHODS}

Guided mode resonance arises from resonant coupling between external radiation and the modes of a photonic crystal slab and is manifest as a spike in the reflectivity signal. ${ }^{5,7}$ The model widely used in the literature for the analysis of guided resonance is derived from phenomenological considerations based on a line shape analysis of the resonance. Indeed, a typical guided-mode reflection spectrum can be considered as an example of the Fano resonance phenomenon, which appears in the optical transmission and reflection spectra of a wide variety of structures, such as metallic or dielectric gratings. ${ }^{21-23}$ In this case, the spectrum consists of an overlapping between a Lorentz resonance shape and a Fabry-Perot background. However, this simple fit approach cannot provide information about the origin of the coupling process between the external radiation and the photonic crystal structure. In Ref. 24, we experimentally demonstrated that, in the range of negative effective refractive indices, a photonic crystal can be modeled as a Lorentz resonator and can exhibit plasmon-like material behavior. $^{25}$ Therefore, we assume that the PhC slab can be described by a dielectric function given by a simple Lorentz function:

$$
\varepsilon(\omega)=\frac{f \omega_{0}^{2}}{\omega_{0}^{2}-\omega^{2}+i \gamma \omega}+\varepsilon_{\infty}
$$




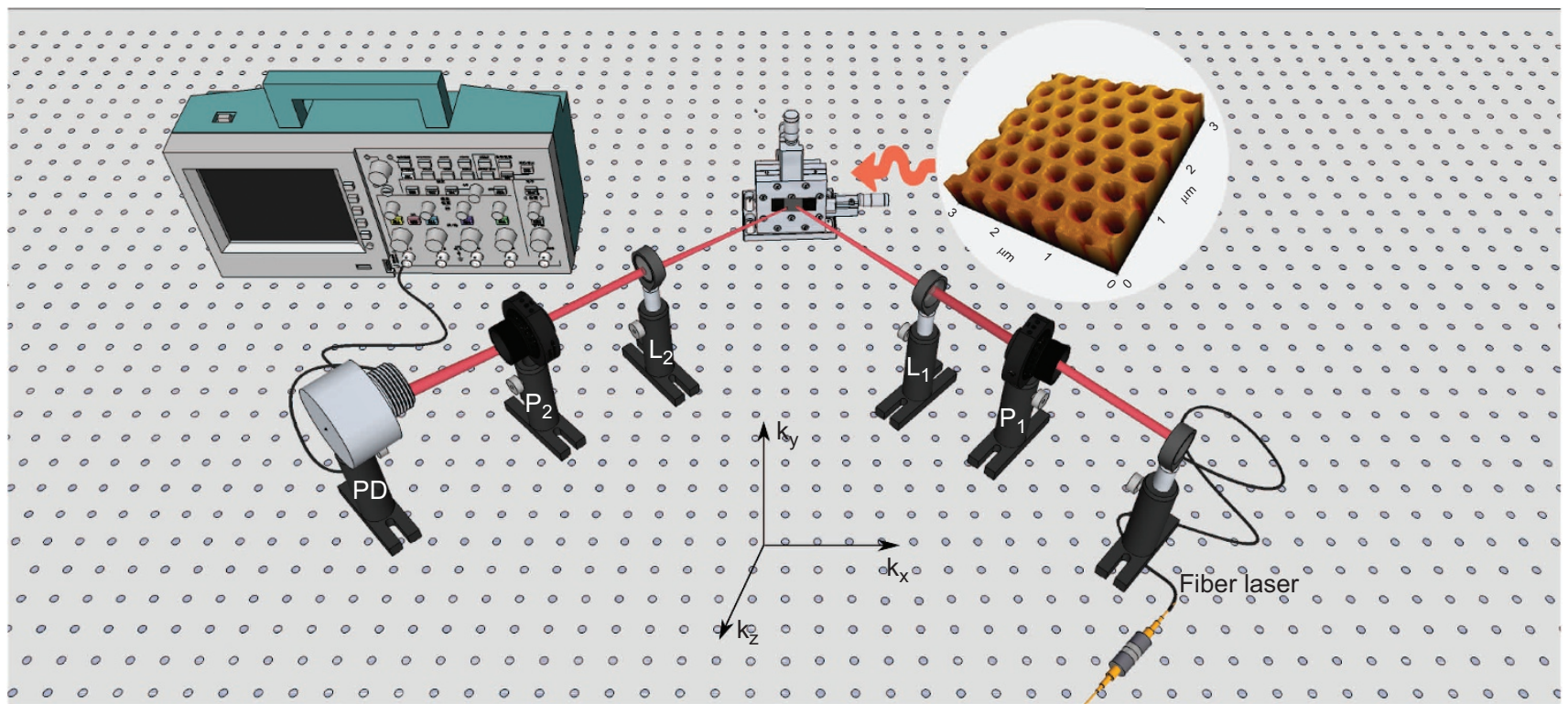

Figure 1 Schematic view of the experimental set-up used to couple laser light (tunable diode laser, 1520-1620 nm) into the photonic crystal. The inset shows an AFM image of the photonic crystal sample. Please note that the shape of the holes results from the AFM probe effect. AFM, atomic force microscopy; L, lens; P, polarizer; PD, photodiode.
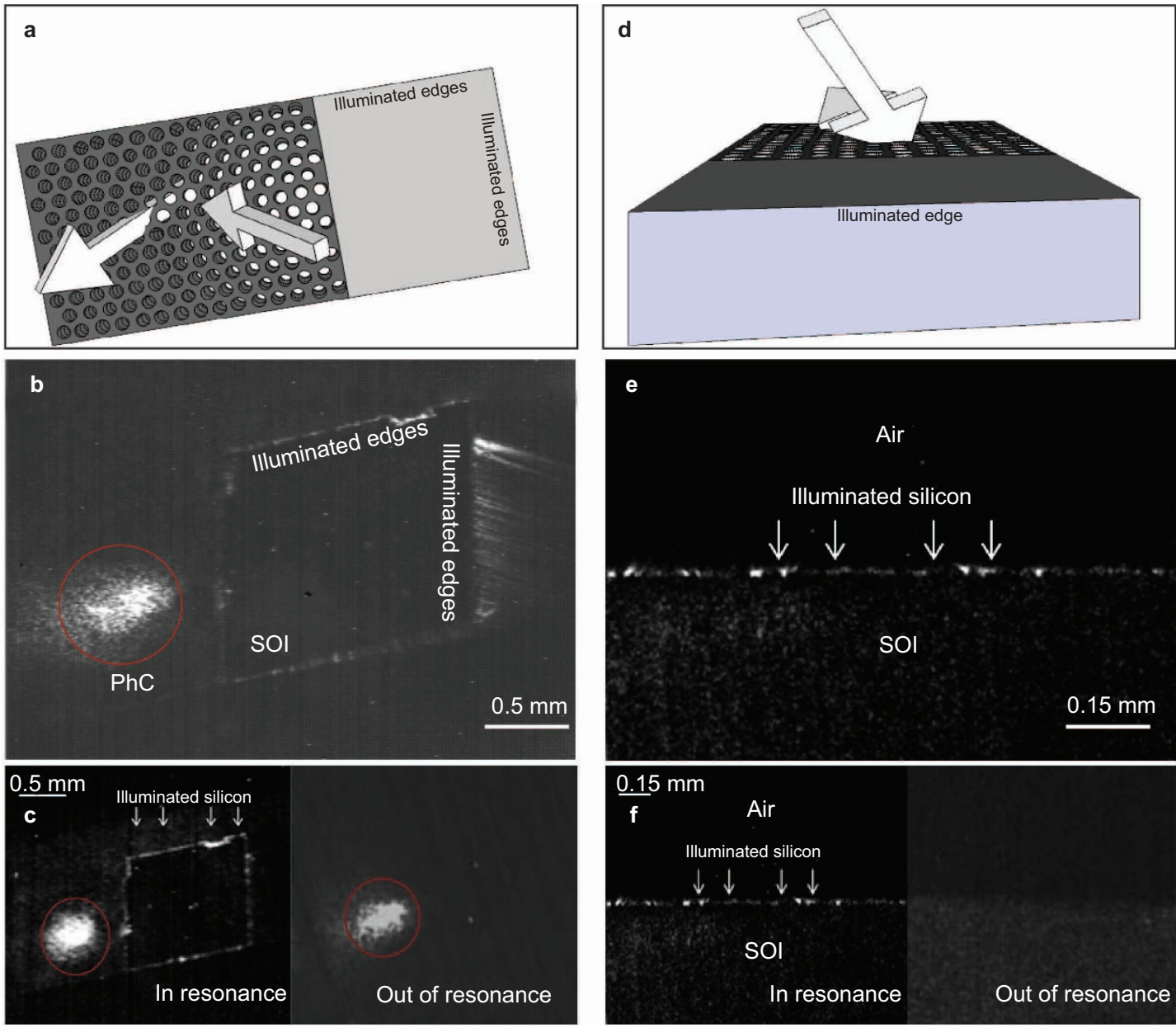

Figure 2 Sketches of the IR camera positions (a, $\mathbf{d})$ and infrared images of the illuminated SOI region when resonance occurs (b,e). Comparison between IR images in the resonance condition $\left(\lambda=1588 \mathrm{~nm}\right.$ and $\left.\theta=65^{\circ}\right)$ and in the out-of-resonance condition $\left(\lambda=1592 \mathrm{~nm}\right.$ and $\left.\theta=65^{\circ}\right)$ acquired in front of the sample (c) and from the lateral side (f). The red circle indicates the spot at which the laser is incident on the sample. IR, infrared; SOI, silicon-on-insulator. 
where $\varepsilon$ is the dielectric constant, $\omega_{0}$ is the resonant frequency and $\gamma$ is the damping constant. In this way, the resonator behavior is inherent to the material, and the $\mathrm{PhC}$ is described as a homogenous material with $\varepsilon(\omega)$ given by Equation (1). In such a case, we can use the usual Fresnel relations to study the features of guided resonance in the reflectivity spectrum. For a three-layer system of dielectric 1 /photonic crystal/dielectric 3, the reflectivity $R$ for p-polarized light is given by Raether $^{4}$

$$
R=\left|r_{123}^{p}\right|^{2}=\left|\frac{r_{12}^{p}+r_{23}^{p} \exp \left(2 i k_{z 2} d\right)}{1+r_{21}^{p} r_{23}^{p} \exp \left(2 i k_{z 2} d\right)}\right|^{2}
$$

with the reflection coefficients given by

$$
r_{i j}^{p}=\frac{\varepsilon_{j} k_{z i}-\varepsilon_{i} k_{z j}}{\varepsilon_{j} k_{z i}+\varepsilon_{i} k_{z j}}
$$

and the wave vector components along $z$ axis defined as

$$
k_{z i}=\sqrt{\varepsilon_{i} \frac{\omega^{2}}{c^{2}}-\frac{\omega^{2}}{c^{2}} \sin ^{2} \theta}
$$

The photonic crystal sample is a slab composed of a hexagonal lattice with a thickness of $0.7 \mu \mathrm{m}$, as shown in Figure 1. The photonic lattice was obtained using a high-precision nanofabrication process based on high-voltage electron beam lithography and a gas chopping inductively coupled plasma etching process, which alternates an etching step using $\mathrm{SF}_{6}$ and Ar with a passivation step using $\mathrm{CHF}_{3}$ and $\mathrm{CH}_{4}$. Starting with a silicon-on-insulator (SOI) wafer $(1.5 \mu \mathrm{m}$ silicon layer on top of a $1 \mu \mathrm{m}$ oxide layer), we spin on ZEP 520, a positive electron beam resist, at a thickness of $370 \mathrm{~nm}$. The resist is patterned using a Vistec VB300UHR EWF electron-beam lithography system and is developed with n-amyl acetate. The electron beam-patterned resist is used as a mask to etch the underlying silicon layer, down to the SOI, in an Oxford Plasmalab 100 ICP-RIE with a resist mask. The $\mathrm{SF}_{6}$ chemistry provides the free radicals for isotropic Si etching, while $\mathrm{O}_{2}$ promotes the growth (at cryogenic temperatures) of a passivation film. This passivation film, with a generic temperature-sensitive formula of $\mathrm{Si}_{x} \mathrm{~F}_{y} \mathrm{O}_{z}$, protects the side walls of the etched structures and evaporates after the sample warms to room temperature, leaving behind a clean surface. The etching was performed at $-120{ }^{\circ} \mathrm{C}$. The resulting pattern is formed of cylindrical air holes, and the device is characterized by a lattice constant $a=472 \mathrm{~nm}$, a hole radius $r=0.385 a$ and an area of $1 \times 1 \mathrm{~mm}^{2}$. The ratio $r / a=0.385$ guarantees the condition of a negative index medium $\overrightarrow{\mathbf{v}} \cdot \overrightarrow{\mathbf{g}}<0 .{ }^{14-18,20,25,26}$ In this case, the photonic crystal behaves as a medium with an effective isotropic index $n_{\mathrm{eff}}=-1$ for a wavelength of $1.55 \mu \mathrm{m}$. Experimental reflectivity spectra from the photonic crystal sample were obtained using a tunable CW diode laser (Ando AQ4321D) that emits monochromatic light with a maximum variable power of $5 \mathrm{~mW}$ and a wavelength varying between $1520 \mathrm{~nm}$ and $1620 \mathrm{~nm}$. Because the guided mode resonance is polarization-dependent, ${ }^{5,7}$ the radiation is linearly polarized and is then focused at the top surface of the sample. The light reflected from the sample is polarized again and is detected by a photodiode (Thorlabs high-speed InGaAs DET410). The incidence angle was varied from $40^{\circ}$ to $75^{\circ}$. The experimental set-up is schematized in Figure 1.

\section{RESULTS AND DISCUSSION}

As previously illustrated, a sharp peak appears in the reflection spectrum when coupling between the incident radiation and a guided mode occurs. This resonance has only been observed for p-polarization, i.e., with the electric field vector parallel to the incident plane. Although guided resonance has been studied for many years, there have been no experimental reports concerning the propagation of coupled light through the photonic crystal, and there is a gap in research on which modes are excited inside the structure. Whenever coupling occurs, we assume that the radiation propagates through the photonic crystal and can be detected by the out-of-plane scattering from the surface. Figure 2 shows infrared (IR) images of the radiation coupled into the structure under resonance conditions $\left(\lambda=1588 \mathrm{~nm}\right.$ and $\left.\theta=65^{\circ}\right)$ acquired from front of the slab and the lateral side. Surprisingly, the radiation propagates for a few millimeters into the photonic crystal. In correspondence to the guided resonance, the light passes through the entire photonic crystal $\left(1 \times 1 \mathrm{~mm}^{2}\right)$, passes through the adjacent SOI planar waveguide (approximately $1 \mathrm{~mm}$ in length) and is then scattered at the end of the SOI region due to the irregularities of the surface, illuminating the whole perimeter of the right SOI region, as shown in Figure $2 \mathrm{~b}$ and 2e. When the wavelength is changed to be slightly out of resonance $(\lambda=1591 \mathrm{~nm})$, the light does not couple with the structure and the SOI perimeter is no longer illuminated (see Figure $2 \mathrm{c}$ and $2 \mathrm{f}$ for a comparison of the resonance and out-of-resonance conditions). Interestingly, backpropagation of the coupled radiation was observed, which can be related to the negative index behavior of the photonic crystal under consideration. This effect was confirmed by placing an IR camera on the positive refraction side of the device, where no radiation was detected. Rigorous coupled wave approach simulations provided further information regarding this phenomenon. The calculated time-averaged Poynting vector inverts its direction while crossing the photonic crystal
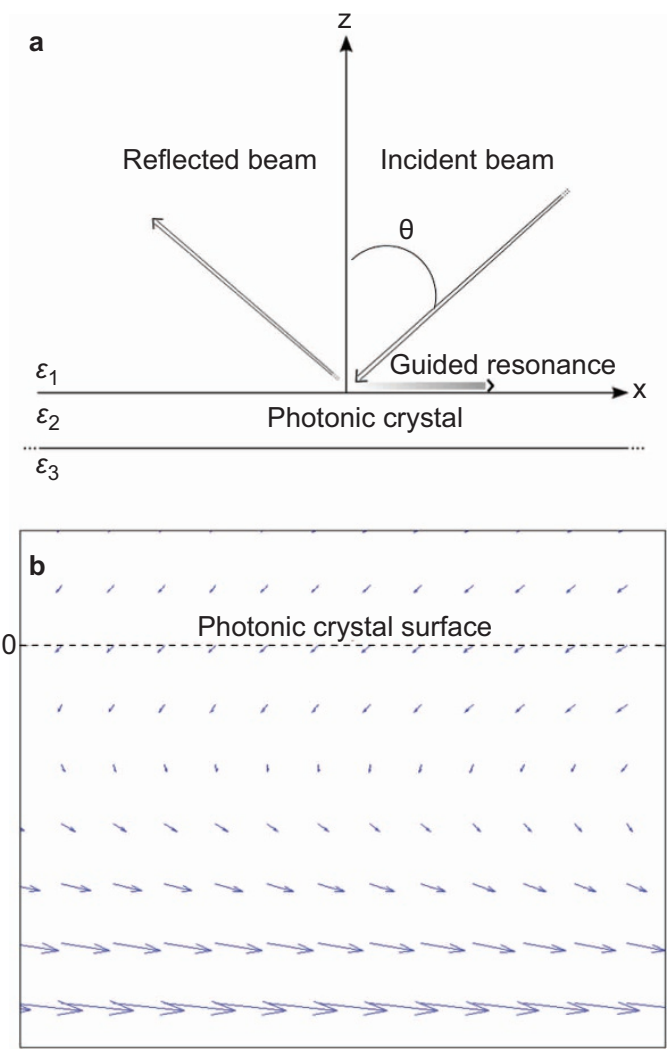

Figure 3 (a) A sketch of the incident, reflected and guided beams. (b) The calculated time-averaged Poynting vector in a $60 \times 60-\mathrm{nm}^{2}$ area in the $x-z$ plane, crossing the $\mathrm{PhC}$ surface under the resonance conditions. $\mathrm{PhC}$, photonic crystal. 
interface within a few tens of nanometers, as shown in Figure 3. Finally, we validate our new theoretical approach by directly fitting the experimental spectrum with the Fresnel formula (2). The calculated fit curve shows excellent agreement with the experimental spectrum, as reported in Figure 4a. The fitted Lorentz parameters are $f=0.0007$, $\gamma=-1.5 \times 10^{11} \mathrm{~s}, \varepsilon_{\infty}=12$ in the resonance condition, $\lambda_{0}=1588 \mathrm{~nm}$ and $\theta=65^{\circ}$. These parameters remain the same as the incident angle changes, excepting for the resonant frequency $\omega_{0}$, which changes following the band structure, as shown in Figure $4 \mathrm{~b}$. This result confirms that the negative index $\mathrm{PhC}$ layer is strongly correlated with a resonant effective permittivity, ${ }^{27}$ which, in this case, can be modeled with a Lorentzian function (1). ${ }^{24}$ Moreover, we estimated the experimental quality factor $\mathrm{Q}$ and obtained a value on the order of $10^{3}$, which is among the highest values experimentally determined for a $\mathrm{PhC}$ slab. In addition, by varying the angle of incidence in the reflectivity spectrum, we calculated the band structure of the photonic crystal. ${ }^{5,7}$ In Figure $4 \mathrm{~b}$, the measured dispersion characteristics of the surface modes as a function of the in-plane wavenumber are displayed. Once again, the measured values (squares) are in excellent agreement with the simulated dispersion curve (triangles) obtained from the rigorous coupled wave approach. These values agree with the sign of the calculated and measured group velocity of the mode along the parallel wavevector, $k_{\mathrm{p}}$. Indeed, from Figure $4 \mathrm{~b}$, we find
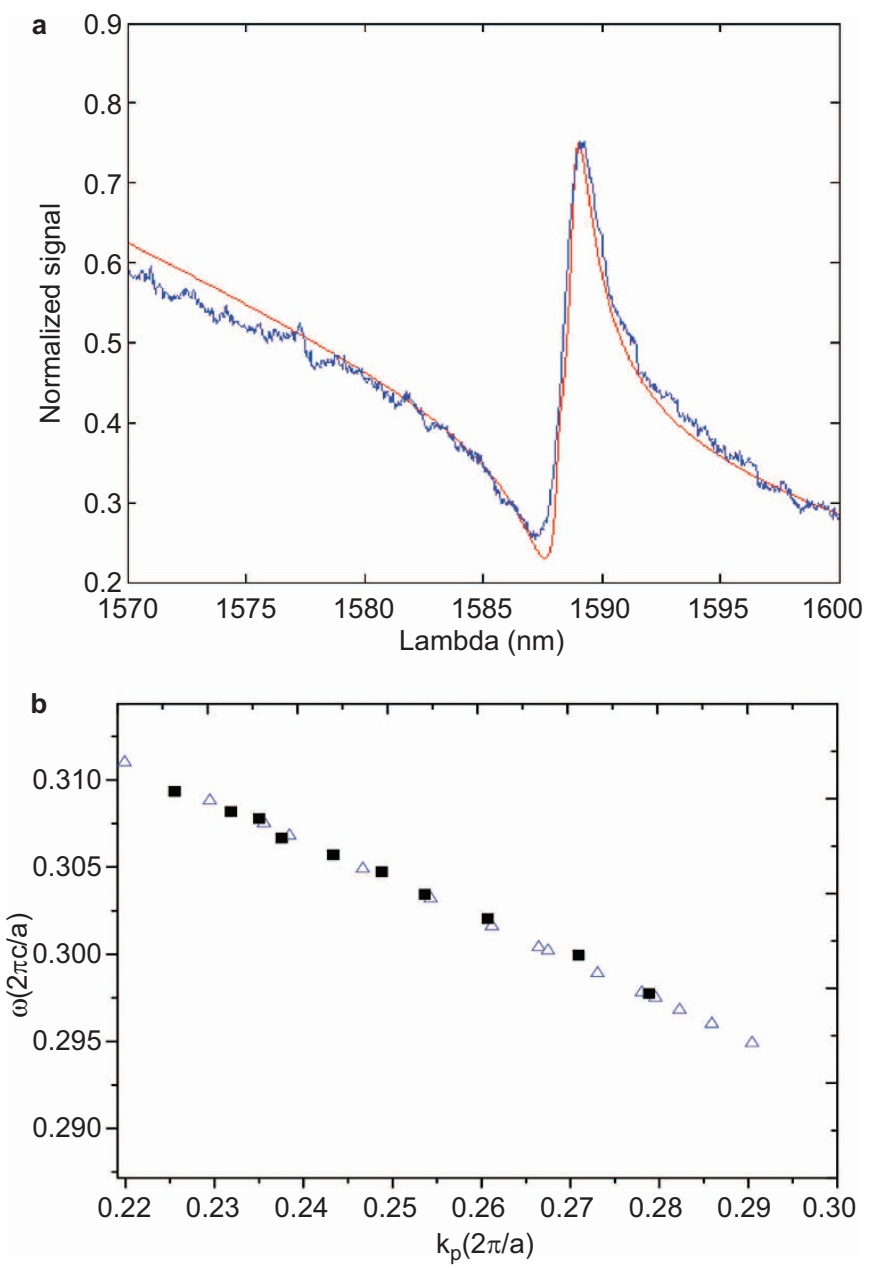

Figure 4 (a) Comparison between theoretical and experimental values. The fitted curve (red line) shows good agreement with the experimental spectrum (blue line). (b) Comparison between the calculated (triangles) and measured (squares) band structures. $\overrightarrow{\mathbf{v}_{g}}=\partial \omega / \partial k_{p}<0$, in agreement with the results obtained from the imaging analysis.

\section{CONCLUSIONS}

This work presents new insight into the coupling of guided resonance in photonic crystal structures. In addition to a study of the reflection spectrum, we presented imaging measurements demonstrating the coupling of external radiation with photonic crystal modes. Furthermore, we succeeded in following the propagation of the guided radiation into the structure, showing that the light backpropagates a few millimeters into the slab. We related this effect to the negative refraction behavior of the photonic crystal and modeled its effective permittivity with a Lorentzian function. Using this model, we were able to fit the experimental spectrum with a simple Fresnel relation, thus improving upon the standard phenomenological line shape analysis.

\section{ACKNOWLEDGMENTS}

Portions of this work were performed at the Molecular Foundry, Lawrence Berkeley National Laboratory, which is supported by the Office of Science, Office of Basic Energy Sciences, of the US Department of Energy under contract no. DE-AC02-05CH11231. This work was partially supported by the Italian Ministry of University and Research under grants PON PANDION 01_00375.

1 Wood RW. On a remarkable case of uneven distribution of light in a diffraction grating spectrum. Philos Mag Ser 1902; 4: 396-402.

2 Rayleigh L. On the dynamical theory of gratings. Proc R Soc Lond Ser A 1907; 79: 399-416.

3 Hessel A, Oliner AA. A new theory of Wood's anomalies on optical gratings. App/ Opt 1965; 4: 1275-1297.

4 Raether H. Surface Plasmons on Smooth and Rough Surfaces and on Gratings. Berlin/ New York: Springer-Verlag; 1988.

5 Astratov VN, Whittaker DM, Culshaw IS, Stevenson RM, Skolnick MS et al. Photonic band-structure effects in the reflectivity of periodically patterned waveguides. Phys Rev B 1999; 60: 16255-16258.

6 Astratov VN, Culshaw IS, Stevenson RM, Whittaker DM, Skolnick MS et al. Resonant coupling of near-infrared radiation to photonic band structure waveguides. J Lightwave Technol 1999; 17: 2050-2057.

7 Astratov VN, Stevenson RM, Culshaw IS, Whittaker DM, Skolnick MS et al. Heavy photon dispersions in photonic crystal waveguides. Appl Phys Lett 2000; 77: 178180.

8 Magnusson R, Wang SS. Multilayer waveguide-grating filters. App/ Opt 1995; 34 2414-2420.

9 Magnusson R, Wang SS. New principles for optical filters. Appl Phys Lett 1992; 61 : 1022-1024

10 Wang S. Two-dimensional distributed-feedback lasers and their applications. App/ Phys Lett 1973; 22: 460-463.

11 Ma J, Martinez L, Povinelli ML. Optical trapping via guided resonance modes in a SlotSuzuki-phase photonic crystal lattice. Opt Express 2012; 20: 6816-6824.

12 Kappel C, Selle A, Bader MA, Marowsky G. Double grating waveguide structures: 350 fold enhancement of two-photon fluorescence applying ultrashort pulses. Sens Actuators B 2005; 107: 135-139.

13 Suh W, Fan S. All-pass transmission or flattop reflection filters using a single photonic crystal slab. Appl Phys Lett 2004; 84: 4905-4908.

14 Notomi M. Theory of light propagation in strongly modulated photonic crystals: refractionlike behavior in the vicinity of the photonic band gap. Phys Rev $B$ 2000; 62: 10696-10705.

15 Mocella V, Cabrini S, Chang A, Dardano P, Moretti L et al. Self-collimation of light over millimeter-scale distance in a quasi-zero-average-index metamaterial. Phys Rev Lett 2009; 102: 133902

16 Notomi M. Negative refraction in photonic crystals. Opt Quantum Electron 2002; 34 : 133-143.

17 Di Caprio G, Dardano P, Coppola G, Cabrini S, Mocella V. Digital holographic microscopy characterization of superdirective beam by metamaterial. Opt Lett 2012; 37: 1142-1144.

18 Mocella V, Dardano P, Moretti L, Rendina I. Influence of surface termination on negative reflection by photonic crystals. Opt Express 2007; 15: 6605-6611.

19 Kocaman S, Chatterjee R, Panoiu NC, McMillan JF, Yu MB et al. Observation of zeroorder band gaps in negative refraction photonic crystal superlattices at near-infrared frequencies. Phys Rev Lett 2009; 102: 203905.

20 Mocella V, Dardano P, Rendina I, Cabrini S. An extraordinary directive radiation based on optical antimatter at near infrared. Opt Express 2010; 18: 25068-25074.

21 Fan S, Joannopoulos J. Analysis of guided resonances in photonic crystal slabs. Phys Rev B 2002; 65: 235112 . 
22 Fan S, Suh W, Joannopoulos JD. Temporal coupled-mode theory for the Fano resonance in optical resonators. J Opt Soc Am A 2003; 20: 569-572.

23 Miroshnichenko A, Flach S, Kivshar YS. Fano resonances in nanoscale structures. Rev Mod Phys 2010; 82, 2257-2298.

24 Dardano P, Gagliardi M, Rendina I, Cabrini S, Mocella V. Ellipsometric determination of permittivity in a negative index photonic crystal metamaterial. Ligh Sci App/2012; 1: e42. doi:10.1038/lsa.2012.42.

25 De Tommasi E, De Luca AC, Cabrini S, Rendina I, Romano S et al. Plasmon-like surface states in negative refractive index photonic crystals. Appl Phys Lett 2013; 102: 081113.
26 Chatterjee R, Panoiu NC, Liu K, Dios Z, Yu MB et al. Achieving subdiffraction imaging through bound surface states in negative refraction photonic crystals in the nearinfrared range. Phys Rev Lett 2008; 100: 187401.

27 Vynck K, Felbacq D, Centeno E, Căbuz AI, Cassagne D et al. All-dielectric rod-type metamaterials at optical frequencies. Phys Rev Lett 2009; 102: 133901.

(c) (i) (2) This work is licensed under a Creative Commons Attribution-

BY NC SA NonCommercial-ShareAlike 3.0 Unported license. To view a copy of this license, visit http://creativecommons.org/licenses/by-nc-sa/3.0 